\title{
A parasite indirectly affects nutrient distribution by common mycorrhizal networks between host and neighboring plants
}

\author{
YongGe Yuan, ${ }^{1,2}$ Mark van Kleunen (iD), ${ }^{1,2,3}$ AND JunMin Li (D) 1,2,4 \\ ${ }^{1}$ School of Advanced Study, Taizhou University, Taizhou 318000 China \\ ${ }^{2}$ Zhejiang Provincial Key Laboratory of Plant Evolutionary Ecology and Conservation, Taizhou University, Taizhou 318000 China \\ ${ }^{3}$ Department of Biology, University of Konstanz, Konstanz 78464 Germany
}

Citation: Yuan, Y., M. van Kleunen, and J. Li. 2021. A parasite indirectly affects nutrient distribution by common mycorrhizal networks between host and neighboring plants. Ecology 102(5):e03339. 10.1002/ecy. 3339

Abstract. Cascading effects are ubiquitous in nature and can modify ecological processes. Most plants have mutualistic associations with mycorrhizal fungi, and can be connected to neighboring plants through common mycorrhizal networks (CMNs). However, little is known about how the distribution of nutrients by CMNs to the interconnected plants is affected by higher trophic levels, such as parasitic plants. We hypothesized that parasitism would indirectly drive CMNs to allocate more nutrients to the nonparasitized neighboring plants rather than to the parasitized host plants, and that this would result in a negative-feedback effect on the growth of the parasitic plant. To test this, we conducted a container experiment, where each container housed two in-growth cores that isolated the root system of a single Trifolium pratense seedling. The formation of CMNs was either prevented or permitted using nylon fabric with a mesh width of 0.5 or $25 \mu \mathrm{m}$, respectively. In each container, either both T. pratense plants were not parasitized or only one was parasitized by the holoparasite Cuscuta australis. To quantify the nutrient distribution by CMNs to the host and neighboring plants, we used ${ }^{15} \mathrm{~N}$ labeling. Growth and ${ }^{15} \mathrm{~N}$ concentrations of $C$. australis and $T$. pratense were measured, as well the arbuscular mycorrhizal fungi-colonization rates of $T$. pratense. We found that parasitism by $C$. australis reduced the biomass of $T$. pratense. In the absence of the parasite, $\mathrm{CMNs}$ increased the ${ }^{15} \mathrm{~N}$ concentration of both $T$. pratense plants, but did not affect their biomass. However, with the parasite, the difference between host and neighboring T. pratense plants in ${ }^{15} \mathrm{~N}$ concentration and biomass were amplified by CMNs. Furthermore, CMNs decreased the negative effect of $C$. australis on growth of the host T. pratense plants. Finally, although CMNs did not influence the ${ }^{15} \mathrm{~N}$ concentration of $C$. australis, they reduced its biomass. Our results indicate that when $T$. pratense was parasitized by $C$. australis, CMNs preferentially distributed more mineral nutrients to the nonparasitized neighboring $T$. pratense plant, and that this had a negative feedback on the growth of the parasite.

Key words: arbuscular mycorrhizal fungi; cascading effect; common mycorrhizal networks; host plant; nitrogen; parasitic plant.

\section{INTRODUCTION}

In ecological communities, species are linked to each other in networks of direct and indirect interactions (Hartley et al. 2015, Haan et al. 2018). As a consequence, changes in the presence of some species may have cascading effects at other trophic levels that can indirectly affect ecological processes. Many parasitic plants are generalists that can use many species as hosts, and are known to have profound indirect effects on above- and belowground organisms (e.g., soil microbes, neighboring plants) (Bardgett et al. 2006, March and Watson 2007, Watson 2009, Yang et al. 2019). It has

Manuscript received 12 August 2020; revised 27 November 2020; accepted 11 January 2021. Corresponding Editor: Ken N. Paige.

${ }^{4}$ Corresponding Author. E-mail: lijmtzc@126.com been shown that they can indirectly affect soil microbes and their activities (Bardgett et al. 2006, March and Watson 2007, Li et al. 2008, Watson 2009, Sui et al. 2019). These indirect effects might benefit the neighboring plants, and thus become visible aboveground (Yang et al. 2019). However, whether and, if so, how parasitic plants trigger such cascading effects from host plants, via soil microbes, on neighboring plants is not known.

Parasitic plants are connected to their host via rootlike structures called haustoria (Sui et al. 2014). They extract resources (e.g., water, sugars, nutrients) from the host plants, resulting in depressed growth of the host plant (Sui et al. 2019, Yang et al. 2019). Moreover, the parasite and host can exchange various signals that affect their growth (Westwood and Kim 2017, Banerjee 2020). Because in nature host plants interact with various organisms at other trophic levels, we expect that the 
effect of a parasitic plant on its host indirectly affects the interactions of the host with other organisms, such as soil microbes (Gao et al. 2019, Sui et al. 2019). Most parasitized host plants can still form symbiotic relationships with arbuscular mycorrhizal fungi (AMF), and have common mycorrhizal networks (CMNs) with neighboring plants (Egerton-Warburton et al. 2007, Mikkelsen et al. 2008, Weremijewicz and Janos 2013). CMNs play an important role in plant interactions by regulating the distribution of mineral nutrients between interconnected plant individuals (Egerton-Warburton et al. 2007, Mikkelsen et al. 2008, Weremijewicz and Janos 2013, Workman and Cruzan 2016). However, whether changes in nutrient distribution by CMNs provide a mechanistic link for cascading effects of parasites on neighboring plants of the host plants is not known.

There are three possible patterns (or hypotheses) with regard to how $\mathrm{CMNs}$ distribute nutrients among different plants. The first one is that nutrient distribution by CMNs is more or less random and not correlated with size or competitive ability of the plants. Indeed, Chiariello et al. (1982) found that mineral nutrients taken up by CMNs were "shared" among interconnected plants with no clear pattern as to which individuals received the most mineral nutrients. The second hypothesis is that CMNs preferentially transfer more mineral nutrients to plants suppressed by, for example, shading, thus alleviating the plants from competitive pressures of surrounding individuals (Grime et al. 1987). However, there is little evidence in support of this hypothesis. The third hypothesis is that CMNs transfer mineral nutrients preferentially to large host individuals, which are likely to be strong carbon suppliers, rather than to suppressed individuals that are minor carbon suppliers (Lekberg et al. 2010, Merrild et al. 2013). In line with this idea, Merrild et al. (2013) found that $\mathrm{CMNs}$ allocated more phosphorus to large established plants than to small seedlings, thus amplifying nutrient competition between the connected plants. Similarly, Weremijewicz et al. (2016) showed that CMNs preferentially distributed more mineral nutrients to large, unshaded individuals than to connected small, shaded individuals. The third hypothesis thus seems to have most support.

One possible mechanism by which CMNs may preferentially allocate more nutrients to large, strong plants might be through a "reciprocal rewards" mechanism (Kiers et al. 2011). Kiers et al. (2011) proposed this mechanism based on in vitro research with root-organ cultures, which showed that plants rewarded the most beneficial fungal partners with more resources, such as carbohydrates. In turn, their fungal partners reinforced this cooperation by increasing nutrient transfer to those roots that provided more carbohydrates. Similarly, CMNs might reward the interconnected plants that supply the most resources with more mineral nutrients, so that these plants would continue to provide more carbohydrates to the CMNs.
Parasitic plants solely or partly depend on host plants for carbon. When parasitized host plants are connected with their nonparasitized neighbors via CMNs, it is likely that they cannot provide as much carbohydrate to CMNs as their nonparasitized conspecific neighbors. This is because host plants have already lost part of their photosynthetic products to the parasitic plants. If the "reciprocal rewards" mechanism applies, CMNs might thus preferentially distribute more mineral nutrients to the nonparasitized conspecific neighbors than to the parasitized hosts. Consequently, as growth of the parasitic plant depends on the growth of its host (Zhang et al. 2013), it is likely that the unequal mineral nutrient distribution by CMNs will ultimately be disadvantageous to the growth of the parasitic plant.

In this study, we tested whether there are cascading effects of a parasitic plant on its host plant, and via CMNs on the neighboring plants. Specifically, we assessed the effect of the parasitic plant Cuscuta australis on nutrient distribution by CMNs between parasitized Trifolium pratense and nonparasitized $T$. pratense. We also assessed the potential feedback of the cascading effects on growth of the parasitic plant. We hypothesized that the parasitic plant would indirectly cause CMNs to allocate more mineral nutrients to nonparasitized neighboring plants than to parasitized host plants, and thereby lead to a negative feedback on growth of the parasitic plant.

\section{Methods}

\section{Study species}

Cuscuta australis was used as the parasitic plant species. Cuscuta australis is an obligate shoot parasite, which acquires water, carbon, and nutrients from its host, and thereby inhibits the growth of the host. Cuscuta australis can parasitize a wide range of herbaceous species. As host and neighboring plant species, we chose $T$. pratense, a herbaceous legume, because it is known that $C$. australis heavily suppresses the growth of Trifolium species in nature (Shi et al. 2007), and because $T$. pratense is widely parasitized by $C$. australis in the field.

\section{Experimental containers}

Each of 20 experimental containers $(26 \times 12 \times 12 \mathrm{~cm}$ for length $\times$ width $\times$ height $)$ were filled with $2.5 \mathrm{~kg}$ of sterilized substrate consisting of a 6:3:1 mixture of peat, sand and vermiculite (v:v:v). The peat had a $\mathrm{pH}$ of 6.2 , and contained $635 \mathrm{~g} / \mathrm{kg}$ organic matter, $8.43 \mathrm{~g} / \mathrm{kg}$ total nitrogen, and $0.604 \mathrm{~g} / \mathrm{kg}$ total phosphorus. Two in-growth cores, each filled with $0.5 \mathrm{~kg}$ of the same sterilized substrate, were inoculated with AMF as described below, and dug into the substrate of each container (Fig. 1). The in-growth cores were $9 \mathrm{~cm}$ apart, and were fabricated using $5.5 \times 12 \mathrm{~cm}$ (diameter $\times$ height) PVC cups with openings on the side that 
accounted for a quarter of the total lateral surface area (see Awaydul et al. 2018). Each in-growth core contained either a parasitized or a nonparasitized $T$. pratense plant (Fig. 1). The lateral openings were covered with a nylon fabric with a mesh width of either 0.5 or $25 \mu \mathrm{m}$ (Jiuding High-Tech Filtration Equipment Co., Ltd, Beijing, China). The $0.5-\mu \mathrm{m}$ nylon mesh prevents the penetration by both AMF hypha and plant roots, and the $25-\mu \mathrm{m}$ nylon mesh allows penetration by hyphae but not by roots.

\section{Experimental design}

In each container, one of the two T. pratense plants in an in-growth core was designated to be the host plant, and the other $T$. pratense plant was designated as nonparasitized neighboring plant. The experiment had three factors: (1) the presence or absence of common mycorrhizal networks $(\mathrm{CMN}+, \mathrm{CMN}-)$; (2) the presence or absence of $C$. australis parasitism on the host plant $(\mathrm{P}+$, $\mathrm{P}-$ ); (3) plant status: host or neighboring T. pratense (note that we also refer to one of the T. trifolium plants as host when both plants in a container were nonparasitized). Each treatment combination had five replicate containers.

\section{AMF inoculum}

We conducted a pilot experiment to test whether the experimental methods described by Awaydul et al. (2018) resulted in a CMN between the two T. pratense plants (Appendix S1). In this pilot experiment, we inoculated one of the two plants in each container and examined whether the mycelium colonized the uninoculated plant on the other side of the container. We indeed found that this was the case, no matter whether we inoculated the parasitized host or nonparasitized neighboring plant alone (Appendix S1). To further increase the likelihood that CMNs would establish in the main experiment, we inoculated both $T$. pratense plants in each container. For each in-growth core, $10 \mathrm{~g}$ of an AMF inoculum was mixed into the substrate. The inoculum had been purchased from the Institute of Microbiology, Guangxi Academy of Agricultural Sciences, China, and was derived from pot cultures of Sorghum bicolor (L.) Moench grown in coarse sand. The inoculum consisted of colonized root fragments of S. bicolor, soil, and spores of the AMF Rhizophagus intraradices. This is a widespread generalist AMF species (Moora et al. 2011). The spore density of the AMF inoculum was 168 spores per $10 \mathrm{~g}$. (a) $\quad \mathrm{CMN}-, \mathrm{P}-$

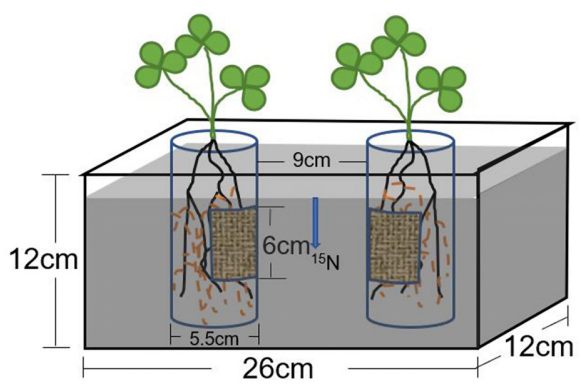

(c) $\mathrm{CMN}-, \mathrm{P}+$

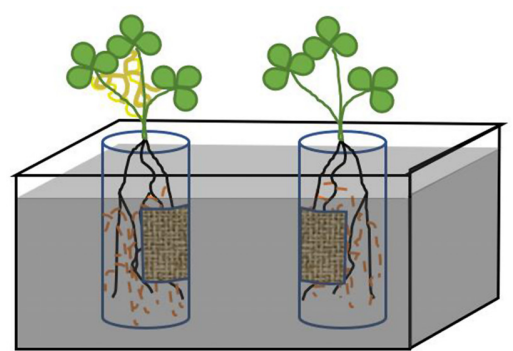

(b)

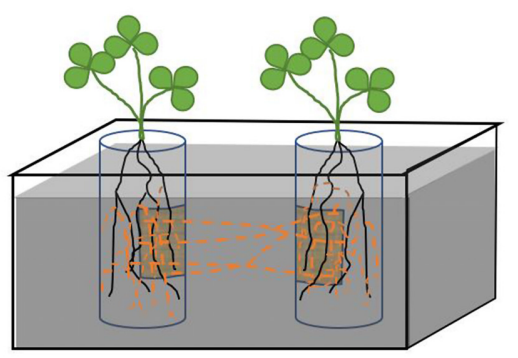

(d)

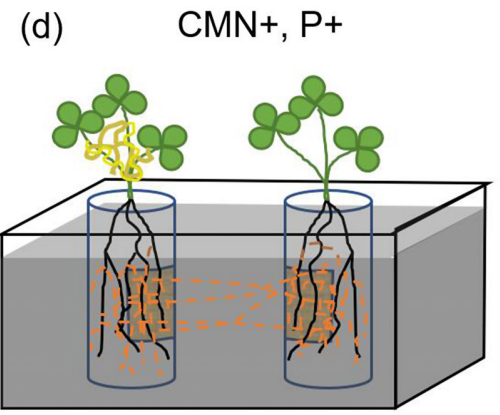

FIG. 1. Diagram showing the experimental design. The paired host and neighboring Trifolium pratense plants colonized by arbuscular mycorrhizal fungi (AMF) were either interlinked through formation of a common mycorrhizal network (CMN) or not, as allowed by lateral side openings in the PVC in-growth cores covered with either a $25-\mu \mathrm{m}$ (permitting) or $0.5-\mu \mathrm{m}$ (preventing) nylon mesh. (a) Absence of CMNs and the parasite, (b) presence of CMNs in the absence of the parasite, (c) absence of CMNs in the presence of the parasite, (d) presence of both CMNs and the parasite. $\mathrm{CMN}-$ : $\mathrm{CMN}$ absent, $\mathrm{CMN}+\mathrm{CMN}$ present, $\mathrm{P}-$ : host $T$. pratense not parasitized by Cuscuta australis, $\mathrm{P}+$ : host $T$. pratense parasitized by $C$. australis. 


\section{Planting, parasitism, and growth conditions}

Seeds of $T$. pratense had been purchased from the Beijing Golden Earth Agricultural Technology Research Institute. On 23 June 2018, seeds of T. pratense were surface sterilized for $10 \mathrm{~min}$ in $5 \%$ sodium hypochlorite and germinated in a mixture of vermiculite and peat moss in the greenhouse facilities of Taizhou University. When seedlings were approximately $2 \mathrm{~cm}$ tall, seedlings of similar size were selected and transplanted individually into the two in-growth cores of each container. Each ingrowth core had been inoculated with AMF on the same day that the seedlings were planted.

Four weeks after transplantation, one 10-cm-long stem piece of $C$. australis was wound counterclockwise around the stems of the host $T$. pratense plants to allow the parasite to infect the plants. Nonparasitized T. pratense plants were used as controls ( $\mathrm{Li}$ et al. 2014). The stems of $C$. australis had been collected from a field population near Taizhou University.

The experiments were conducted in a greenhouse at Taizhou University with a photoperiod of $16 \mathrm{~h}$ and $8 \mathrm{~h}$ of dark at $22^{\circ} \mathrm{C}$ (day) and $18^{\circ} \mathrm{C}$ (night), respectively. Water was sprayed evenly onto the soil of each container every day. We added $20 \mathrm{ml}$ Hoagland nutrient solution to each in-growth core on 13 July 2018.

\section{${ }^{15} \mathrm{~N}$ labeling}

To test the effect of CMNs on nitrogen distribution between host and neighboring $T$. pratense plants, ${ }^{15} \mathrm{~N}$ tracer was introduced $2 \mathrm{wk}$ before the plants were harvested. We dissolved $5 \mathrm{mg}{ }^{15} \mathrm{~N}$-enriched mineral $\left({ }^{15} \mathrm{NH}_{4}\right)_{2} \mathrm{SO}_{4}\left(99.7 \%\right.$ atom ${ }^{15} \mathrm{~N}$; from Shanghai Research Institute of Chemical Industry, Shanghai, China) in $6 \mathrm{ml}$ of distilled water, and injected it with a syringe into the substrate in the middle of each container at a rate of $2 \mathrm{ml}$ per day for $3 \mathrm{~d}$.

\section{Harvest and measurement}

Seventy-seven days after transplantation, the C. australis plants were harvested by detaching them from the host plants. After that, the $T$. pratense plants were separated into shoots and roots by cutting the shoot at a distance of $2 \mathrm{~cm}$ above the soil surface (Göransson et al. 2016). The aboveground biomass was oven dried at $65^{\circ} \mathrm{C}$ for $72 \mathrm{~h}$. The roots were washed to remove soil and carefully checked for the presence of nodules. We found very few nodules, indicating that there was very little contamination with $\mathrm{N}$-fixing rhizobia, which might otherwise have affected our nitrogen concentration results. Half of each root sample, by fresh weight, was then oven dried at $65^{\circ} \mathrm{C}$ for $72 \mathrm{~h}$ to calculate the total root dry biomass. The remaining half of each root sample was used for quantification of AMF colonization using the gridlineintersection method (Giovannetti and Mosse 1980) under an SMZ-168 light microscope ( $\times 20$ magnification,
Motic China Group Co. Ltd., Xiamen, China). A total of $2001-\mathrm{cm}$ root segments were used for each replicate.

The dried shoot samples were ground in a PM400 high-energy ball mill (Retsch Technology GmbH, Haan, Germany) for plant nutrient analysis. Stable $\mathrm{N}$ isotope ratios were determined using a continuous-flow isotoperatio mass spectrometer (CF-IRMS, Thermo Finnigan DELTA Plus, Waltham, Massachusetts, USA). The $\delta^{15} \mathrm{~N}(\%)$ was converted to the absolute isotope ratio $\left({ }^{15} \mathrm{~N} /{ }^{14} \mathrm{~N}\right)$ of the sample based on the atomic ratio of atmospheric nitrogen. The ${ }^{15} \mathrm{~N}$ concentration of the sample was then calculated from fractional abundances $\left({ }^{15} \mathrm{~N} /\left({ }^{15} \mathrm{~N}+{ }^{14} \mathrm{~N}\right)\right)$ and the total $\mathrm{N}$ concentration of the sample (Hu et al. 2001).

\section{Statistical analyses}

The effects of plant status (host vs. neighbor), CMN (present vs. absent) and parasite (present vs. absent), as well as their interactions on aboveground biomass, belowground biomass, AMF-colonization rate, and ${ }^{15} \mathrm{~N}$ concentration of $T$. pratense, were analyzed using mixed-effect models. We accounted for nonindependence of the two $T$. pratense plants in a container by including container identity as the random factor. The effects of CMNs on biomass and ${ }^{15} \mathrm{~N}$ concentration of $C$. australis were analyzed with a one-way analysis of variance (ANOVA). Assumptions of normality and homogeneity of variance were assessed based on standardized residual analysis. Data were transformed as necessary; specifically, a reciprocal transformation for ${ }^{15} \mathrm{~N}$ concentration was conducted. Differences between treatments were compared using the least-significant difference (LSD) at the $5 \%$ significance level. In addition, the relationships of ${ }^{15} \mathrm{~N}$ concentration to aboveground biomass and to AMF-colonization rate were determined using Pearson correlations. The linear mixed-effect models were run in R (3.5.0, R Development Core Team 2018) using the lme function of the nlme package (Pinheiro et al. 2018), and all other analyses were performed using the Statistical Product and Service Solution (SPSS) software (version 16.0; SPSS Inc., Chicago, Illinois, USA).

\section{RESUlts}

\section{Effects of CMNs, the parasite and plant status on Trifolium pratense biomass}

We found that the aboveground biomass of $T$. pratense was significantly affected by plant status $(P=0.005)$ and $\mathrm{CMN}$ presence $(P=0.046$; Table 1$)$. Furthermore, although the parasite did not significantly alter the aboveground biomass on average $(P=0.528)$, there was a significant interactive effect between plant status and infection by the parasite ( $P=0.007$; Table 1$)$. When the designated host $T$. pratense plant was not parasitized by $C$. australis, there was no significant difference in the aboveground biomass between the two 
TABLE 1. Effect of plant status, formation of common mycorrhizal networks (CMNs), and infection by the Cuscuta australis parasite on the aboveground biomass, belowground biomass, arbuscular mycorrhizal fungi (AMF) colonization rate, and ${ }^{15} \mathrm{~N}$ concentration in Trifolium pretense.

\begin{tabular}{|c|c|c|c|}
\hline Effect & df & $F$ & $P$ \\
\hline \multicolumn{4}{|l|}{ Aboveground biomass } \\
\hline Plant status & 1 & 10.516 & 0.005 \\
\hline $\mathrm{CMN}$ & 1 & 4.704 & 0.046 \\
\hline Parasite & 1 & 0.416 & 0.528 \\
\hline Plant status $\times \mathrm{CMN}$ & 1 & 0.560 & 0.465 \\
\hline Plant status $\times$ parasite & 1 & 9.585 & 0.007 \\
\hline $\mathrm{CMN} \times$ parasite & 1 & 3.536 & 0.078 \\
\hline Plant status $\times \mathrm{CMN} \times$ parasite & 1 & 1.075 & 0.315 \\
\hline Denominator & 16 & & \\
\hline \multicolumn{4}{|l|}{ Belowground biomass } \\
\hline Plant status & 1 & 4.814 & 0.043 \\
\hline $\mathrm{CMN}$ & 1 & 0.560 & 0.465 \\
\hline Parasite & 1 & 0.658 & 0.429 \\
\hline Plant status $\times \mathrm{CMN}$ & 1 & 0.590 & 0.454 \\
\hline Plant status $\times$ parasite & 1 & 8.035 & 0.012 \\
\hline $\mathrm{CMN} \times$ parasite & 1 & 2.265 & 0.152 \\
\hline Plant status $\times \mathrm{CMN} \times$ parasite & 1 & 0.353 & 0.561 \\
\hline Denominator & 16 & & \\
\hline \multicolumn{4}{|l|}{ AMF colonization rate } \\
\hline Plant status & 1 & 0.653 & 0.431 \\
\hline $\mathrm{CMN}$ & 1 & 0.032 & 0.861 \\
\hline Parasite & 1 & 0.009 & 0.925 \\
\hline Plant status $\times \mathrm{CMN}$ & 1 & 1.881 & 0.189 \\
\hline Plant status $\times$ parasite & 1 & 5.000 & 0.040 \\
\hline $\mathrm{CMN} \times$ parasite & 1 & 0.002 & 0.968 \\
\hline Plant status $\times \mathrm{CMN} \times$ parasite & 1 & 21.889 & 0.000 \\
\hline Denominator & 16 & & \\
\hline \multicolumn{4}{|l|}{${ }^{15} \mathrm{~N}$ concentration } \\
\hline Plant status & 1 & 4.288 & 0.055 \\
\hline $\mathrm{CMN}$ & 1 & 220.665 & 0.000 \\
\hline Parasite & 1 & 0.412 & 0.530 \\
\hline Plant status $\times \mathrm{CMN}$ & 1 & 8.350 & 0.011 \\
\hline Plant status $\times$ parasite & 1 & 0.648 & 0.433 \\
\hline $\mathrm{CMN} \times$ parasite & 1 & 5.481 & 0.033 \\
\hline Plant status $\times \mathrm{CMN} \times$ parasite & 1 & 8.080 & 0.012 \\
\hline Denominator & 16 & & \\
\hline
\end{tabular}

Bold $P$ values indicate $P<0.05$.

T. pratense plants, and the presence of CMNs did not significantly affect the aboveground biomass of either plants (Fig. 2a). However, when the designated host plants were actually parasitized by $C$. australis, the aboveground biomass of the neighboring plants was significantly higher than that of the host plant (Fig. 2a). Moreover, although the presence of CMNs did not significantly affect the host aboveground biomass, they did significantly increase the aboveground biomass of the neighboring plant (Fig. 2a). In the absence of CMNs, the parasite significantly decreased the aboveground biomass of the host $T$. pratense plants, but had no effect on the neighboring $T$. pratense plants (Fig. 2a). On the other hand, in the presence of CMNs, the parasite did not significantly affect the aboveground biomass of the host $T$. pratense plants, but significantly increased the aboveground biomass of neighboring $T$. pratense plants (Fig. 2a).

Similarly, the belowground biomass of $T$. pratense was significantly affected by plant status $(P=0.043)$, and there was a significant interactive effect between plant status and the presence of the parasite $(P=0.012)$ (Table 1$)$. However, the presence of CMNs and their interactions with plant status, or parasite presence, did not significantly affect the belowground biomass (Table 1). The post hoc test revealed that in the absence of CMNs, the parasite significantly decreased the belowground biomass of the host $T$. pratense plants, but did not significantly affect neighboring $T$. pratense plants (Fig. 2b). In the absence of CMNs, the parasite did not significantly affect belowground biomass of both the host and neighboring T. pratense plants (Fig. 2b). However, in the presence of both CMNs and the parasite, there was a significant difference in belowground biomass between the host and neighboring $T$. pratense plants (Fig. $2 \mathrm{~b}$ ).

\section{Effects of CMNs, the parasite, and plant status on AMF colonization of Trifolium pratense}

For AMF-colonization rate, the main effects of plant status, CMN presence, and parasite presence were not significant $(P>0.05)$, but there was a significant threeway interaction (Table 1). When the designated host T. pratense was not parasitized by $C$. australis, there was no significant difference in AMF-colonization rate of the two T. pratense plants, regardless of the presence of CMNs between the plants (Fig. 2c). However, when the designated host plants were parasitized by $C$. australis in the presence of $\mathrm{CMNs}$, the AMF-colonization rate of the neighboring $T$. pratense plants was significantly higher than that of the host plants (Fig. 2c).

\section{Effects of CMNs, the parasite, and plant status on ${ }^{15} \mathrm{~N}$ concentration of Trifolium pratense}

The ${ }^{15} \mathrm{~N}$ concentration of $T$. pratense shoots was significantly affected by the presence of CMNs $(P<0.001)$ and their interaction with plant status $(P=0.011$; Table 1). Although the parasite did not significantly affect the ${ }^{15} \mathrm{~N}$ concentration of $T$. pratense $(P=0.530)$, there was a significant interactive effect between presence of CMNs and the presence of the parasite $(P=0.033)$, and a significant three-way interactive effect of plant status, presence of CMNs and presence of the parasite $(P=0.012$; Table 1$)$. When the designated host $T$. pratense plant was not parasitized by $C$. australis, there was no significant difference in the ${ }^{15} \mathrm{~N}$ concentration between the two T. pratense plants (Fig. 2d). Presence of CMNs significantly increased the ${ }^{15} \mathrm{~N}$ concentration in both host and neighboring plants (Fig. 2d). However, when host plants were parasitized by $C$. australis in the presence of CMNs, the ${ }^{15} \mathrm{~N}$ concentration of the neighboring $T$. pratense plants was 

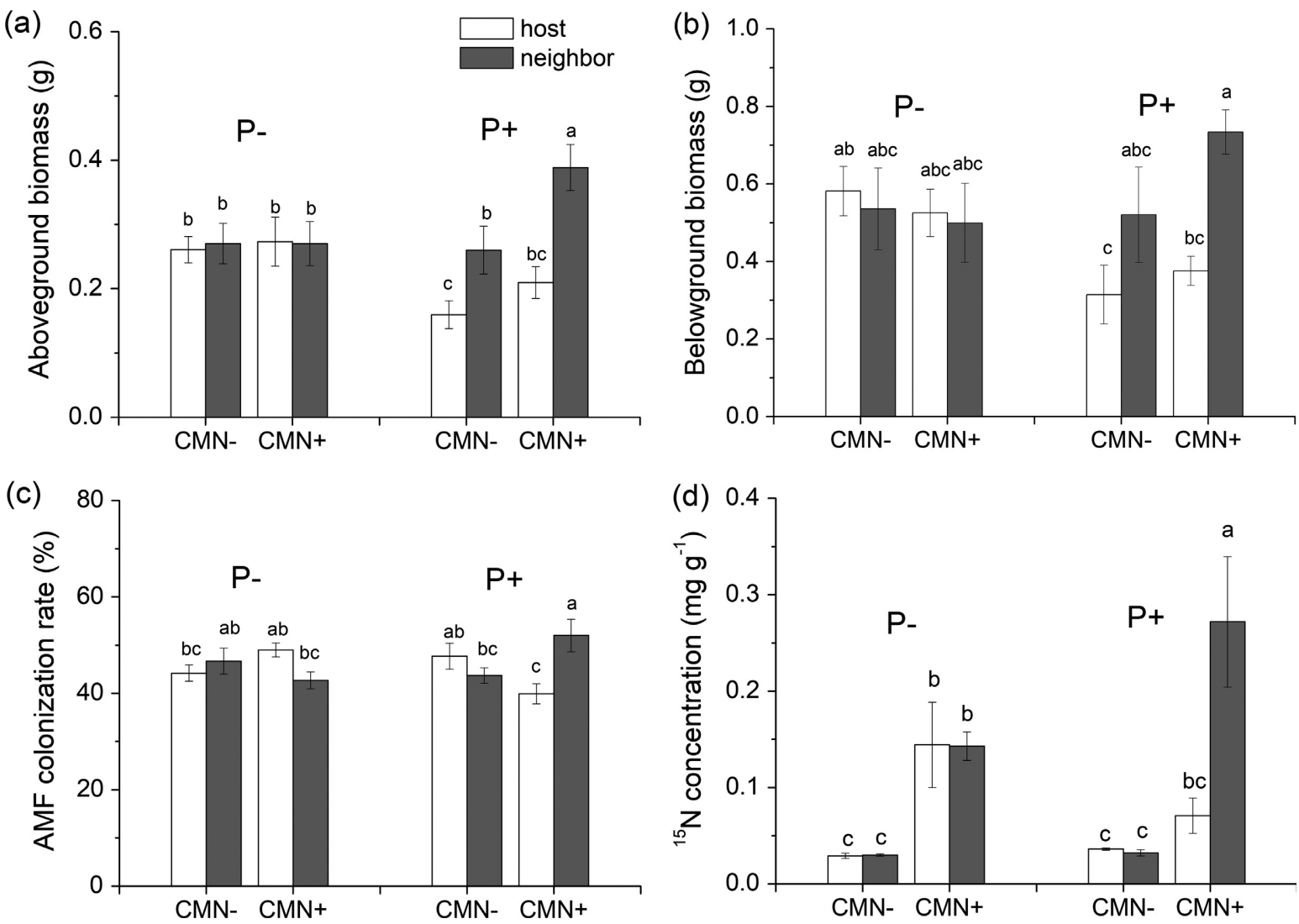

FIG. 2. Effects of plant status (host vs. neighbor), common mycorrhizal networks (CMNs), and Cuscuta australis parasitism on (a) aboveground biomass, (b) belowground biomass, (c) arbuscular mycorrhizal fungi (AMF) -colonization rate, and (d) ${ }^{15} \mathrm{~N}$ concentration of Trifolium pratense. Bars with the same letter do not differ significantly according to a least significant difference (LSD) post hoc test at the $5 \%$ level. Values are means $(n=5) \pm \mathrm{SE}$. CMN-: CMN absent, CMN+: CMN present, $\mathrm{P}-$ : host T. pratense not parasitized by $C$. australis, $\mathrm{P}+$ : host $T$. pratense parasitized by $C$. australis.

significantly higher than for the host $T$. pratense plant (Fig. 2d). Moreover, the presence of CMNs significantly increased the ${ }^{15} \mathrm{~N}$ concentration of the neighboring plants, but had no significant impact on the ${ }^{15} \mathrm{~N}$ concentration of the host plants (Fig. 2d).

In the presence of CMNs, the aboveground biomass was significantly correlated with the ${ }^{15} \mathrm{~N}$ concentration of T. pratense $(r=0.547, \quad P=0.013$; Appendix S1: Fig. S1a). The ${ }^{15} \mathrm{~N}$ concentration of $T$. pratense was not significantly correlated with the AMF-colonization rate $(r=0.227, P=0.336$; Appendix S1: Fig. S1b).

\section{Effect of CMNs on biomass and ${ }^{15} N$ concentration of Cuscuta australis}

The presence of CMNs significantly decreased the biomass of the parasitic plant C. australis (Fig. 3a), but did not significantly affect its ${ }^{15} \mathrm{~N}$ concentration (Fig. 3b).

\section{DisCUSSION}

Our study is, to the best of our knowledge, the first to demonstrate a multitrophic cascading effect triggered by a parasitic plant. By using a stem parasite and isolated root systems, it was possible to assess the effects of the parasite on the host plant, on the CMNs, and ultimately on the neighboring plant. We found that with $C$. australis parasitism, the biomass and ${ }^{15} \mathrm{~N}$ concentration of the neighboring $T$. pratense plants were significantly higher than those of the host $T$. pratense plants. Furthermore, we found that the presence of CMNs decreased the suppression of the host T. pratense biomass by C. australis parasitism, and also decreased the biomass of the parasite. Our results provide evidence for the idea that nutrients in CMNs are preferentially transported to larger, more robust plants rather than to smaller, less robust plants (Merrild et al. 2013, Awaydul et al. 2018). Our results also provide evidence that $C$. australis parasitism can affect plant-plant interactions and create competitive hierarchies between host and neighboring plants (Watson 2009, Poulin 2010). Thus, our results support our hypothesis that $C$. australis parasitism indirectly triggers the CMNs to allocate more mineral nutrients to the nonparasitized neighboring $T$. pratense plants than to parasitized host $T$. pratense plants. This unequal nutrient distribution also resulted in a negative 

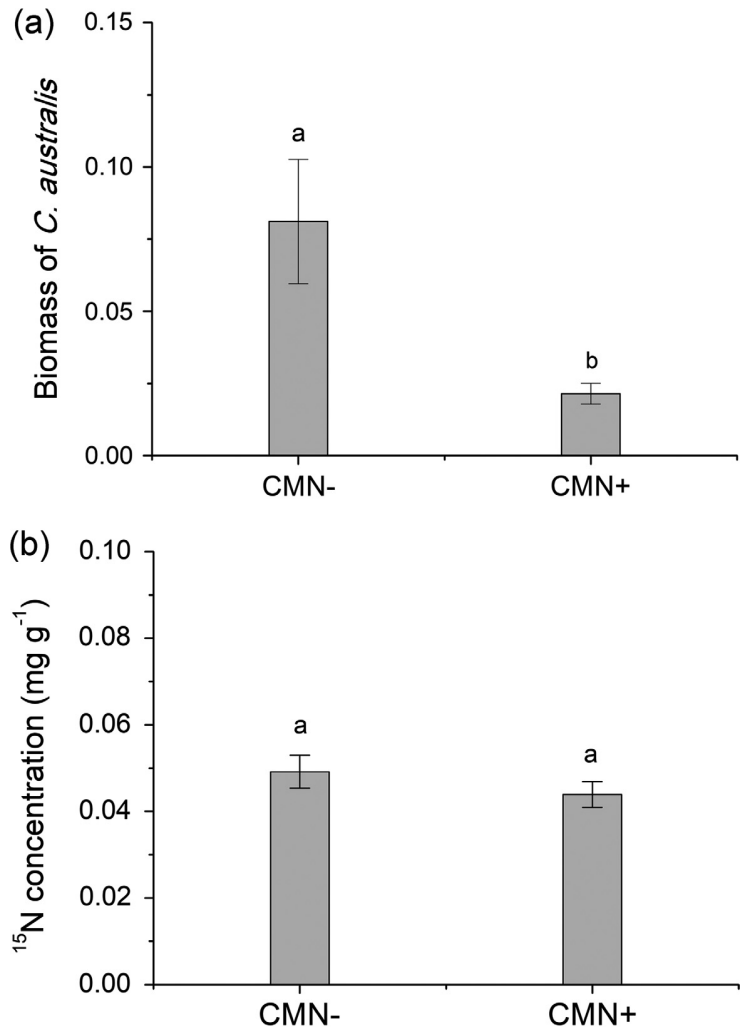

FIG. 3. Effects of common mycorrhizal networks (CMNs) on (a) biomass and (b) ${ }^{15} \mathrm{~N}$ concentration of the parasitic plant Cuscuta australis. Bars with the same letter do not differ significantly according to a least significant difference (LSD) post hoc test at the $5 \%$ level. Values are means $(n=5) \pm \mathrm{SE}$. CMN-: $\mathrm{CMN}$ absent, $\mathrm{CMN}+\mathrm{CMN}$ present.

feedback on the growth of the $C$. australis parasitic plant.

\section{Indirect effect of Cuscuta australis parasitism on nutrient distribution of $C M N S$}

Our results show that in the presence of CMNs and the parasitic plant $C$. australis, the ${ }^{15} \mathrm{~N}$ concentration and biomass of the nonparasitized neighboring T. pratense plants was significantly higher than for the parasitized host $T$. pratense plants (Fig. 2; Appendix S1: Fig. S2). The AMF-colonization rate of neighboring T. pratense plants was also higher than for the host T. pratense plants. However, the AMF-colonization rate did not induce the higher ${ }^{15} \mathrm{~N}$ concentration in the neighboring $T$. pratense plants, as it was not significantly correlated with ${ }^{15} \mathrm{~N}$ concentration in $T$. pratense plants (Appendix S1: Fig. S1).

Cuscuta australis parasitism affected the biomass, AMF colonization, and ${ }^{15} \mathrm{~N}$ concentration of the neighboring $T$. pratense plants only when the CMNs were present. This shows that CMNs are a potential mediator of the indirect interactions between plants. Because $C$. australis is an obligate shoot parasite, it was not in direct contact with the neighboring $T$. pratense plants. This means that the CMNs formed by the AMF-hyphal bridges between the parasitized host and neighboring T. pratense plants were the only possible links for their interaction. Therefore, we conclude that the higher ${ }^{15} \mathrm{~N}$ concentration found in neighboring $T$. pratense compared with the parasitized host $T$. pratense plants in the presence of both CMNs and $C$. australis was mediated through the AMF mycelia that connected the two plants.

Furthermore, our results indicate that CMNs preferentially allocated more nutrients to nonparasitized neighbors than to parasitized host $T$. pratense plants. Such unequal nutrient distribution between plants connected by CMNs has also been reported previously (Walder et al. 2012, Weremijewicz and Janos 2013, Weremijewicz et al. 2016, 2018). There is increasing evidence that the observed unequal nutrient distribution by CMNs does not occur randomly, but rather that CMNs preferentially allocate more nutrients to the most robust plants (Weremijewicz et al. 2016, Awaydul et al. 2018). Consistent with this, our study demonstrates that CMNs preferentially allocated more nitrogen to the larger, nonparasitized neighboring $T$. pratense plants than to the smaller, parasitized $T$. pratense plants, a situation likely driven by the proposed "reciprocal rewards" mechanism (Weremijewicz et al. 2016). In other words, CMNs might allocate more nutrients to nonparasitized plants, thereby most likely enabling the fungus to receive more carbohydrates from these plants in return (Kiers et al. 2011, Jiang et al. 2017). However, whether CMNs are indeed rewarded with more carbohydrates by nonparasitized individuals still needs to be verified. Further studies using ${ }^{13} \mathrm{C}$ labeling to trace the distribution of carbohydrates through the AMF-plant networks are necessary.

Although our pilot experiment indicates that our experimental setup allowed for the establishment of CMNs (Appendix S1), our setup may have resulted in an unintended side effect. Namely, the $0.5-\mu$ m nylon mesh used to prevent CMNs also prevented the plants' AMF from fully accessing the soil compartment. Indeed, the overall low retrieval of ${ }^{15} \mathrm{~N}$ in all plants in the CMN treatment indicates that those plants (and their AMF) had limited access to the soil nutrients. Therefore, an alternative explanation for our findings could be that $C$. australis parasitism decreased nutrient uptake by the AMF-hyphal system of host $T$. pratense plants, and that as consequence there were more nutrients available to the AMFhyphal system of neighboring $T$. pratense plants. Indeed, our results showed that the dominance of the neighboring plants became manifest only in the parasitism treatment with CMNs (i.e., in which the AMF also had full access to the nutrients in the soil compartment). However, we did not find that parasitism significantly reduced the biomass and ${ }^{15} \mathrm{~N}$ concentration of the host plants in the presence of CMNs. The latter suggests that parasitism had no or only a minor effect on the ability of the host plant's AMF to compete for nutrients. It also suggests that the increased biomass and ${ }^{15} \mathrm{~N}$ concentration of the 
neighboring plants in the same containers were unlikely a result of the increased nutrient availability to the AMFhyphal system of neighboring $T$. pratense plants. Therefore, even though the unintended side effect of the increased nutrient availability to the AMF-hyphal system in the presence of CMN may have contributed to our findings, it is unlikely to be the main cause.

\section{Effect of CMNs on Cuscuta australis parasitism}

A limited number of studies have reported that AMF can alleviate damage to host plants by suppressing the growth of parasitic plants, because AMF competes with the parasitic plants for host-derived resources (Tesitel et al. 2011, Li et al. 2012, Sui et al. 2019), especially carbon ( $\mathrm{Li}$ et al. 2019). To the best of our knowledge, our study provides the first direct evidence that the growth of parasitic plants is also inhibited by the presence of CMNs. We found that although CMNs did not significantly affect the ${ }^{15} \mathrm{~N}$ concentration in the parasite $C$. australis, they significantly decreased the $C$. australis biomass. Because $C$. australis is an obligate shoot parasite, the feedback inhibitory effect on the biomass of C. australis mediated by the CMNs could only be induced by the host $T$. pratense plant. However, in the presence of the parasite, CMNs did not have a significant positive impact on the biomass and ${ }^{15} \mathrm{~N}$ concentration of the parasitized host. Although this could be because of the relatively low statistical power of this study, there are also two potential biological reasons that could explain this observation. First, the CMNs probably decreased the mineral nutrient distribution to the parasitized host T. pratense plants, which had a negative effect on the growth of the hosts. Second, it might be directly related to the effect of the parasite $C$. australis. Studies have shown that the growth of parasitic plants is generally positively correlated with the growth of the host plant (Zhang et al. 2013). The negative reallocation of resources by CMNs to host plants probably suppressed the growth of C. australis, which might trigger a feedback loop that has a positive effect on the growth of $T$. pratense. Therefore, we speculate that the direction and strength of the cascading effects triggered by stem parasitic plants and the feedback loop might not be fixed but change over time. More experiments over longer time periods should be done to test how changes in nutrient distribution by $\mathrm{CMNs}$ are triggered by parasitic plants, including tests of how nutrient distribution between nonconspecific plants is affected by different parasitism levels.

\section{Effect of Cuscuta australis parasitism on plant interactions}

Although an increasing number of studies have reported that parasitic plants might change vegetation structure by affecting plant interactions and creating competitive hierarchies between host and neighboring plants (Watson 2009, Poulin 2010, Yang et al. 2019), few of them have revealed the underlying mechanism. Our study provides evidence that parasitic plants might mediate plant interactions between hosts and neighbors by indirectly affecting the nutrient distribution of CMNs. We found that the presence of CMNs amplified the difference in biomass between the parasitized host and nonparasitized neighbor. This result is consistent with those of a previous study showing that parasitism by Cuscuta species strongly affects the composition of soil microbes and resulted in different feedbacks on host and nonhost neighboring plants, thereby shifting competitive hierarchies (Yang et al. 2019). As most plants in nature are likely to be connected by hyphal networks, parasitic plants might shape vegetation structure by the cascading effects mediated by CMNs.

\section{CONCLUSIONS}

In summary, we found that growth suppression of the host by the parasite resulted in the CMNs distributing more of the mineral nutrients to neighboring plants and less to host plants. This change in mineral nutrient distribution in turn resulted in a negative feedback effect on growth of the parasitic plant. Although there are more and more studies indicating that parasitic plants have major indirect effects on the structure and functioning of above- and belowground ecosystems, few studies have revealed the mechanisms. Our results show for the first time how a parasitic plant indirectly affects the nutrient distribution by common mycelial networks between the parasitized host and non-parasitized neighboring plants. We thus provide an example of how a parasitic plant can trigger a series of cascading effects between the aboveand belowground ecosystems.

\section{ACKNOWLEDGMENTS}

The research was financially supported by the Ten Thousand Talent Program of Zhejiang Province (2019R52043) and the National Natural Science Foundation of China (NSFC 30800133).

\section{Literature Cited}

Awaydul, A., W. Zhu, Y. Yuan, J. Xiao, H. Hu, X. Chen, R. T. Koide, and L. Cheng. 2018. Common mycorrhizal networks influence the distribution of mineral nutrients between an invasive plant, Solidago canadensis, and a native plant, Kummerowa striata. Mycorrhiza 29:29-38.

Banerjee, A. 2020. Inter-plant communication via parasitic bridging. Journal of Experimental Botany 71:749-750.

Bardgett, R. D., R. S. Smith, R. S. Shiel, S. Peacock, J. M. Simkin, H. Quirk, and P. J. Hobbs. 2006. Parasitic plants indirectly regulate below-ground properties in grassland ecosystems. Nature 439:969-972.

Chiariello, N., J. C. Hickman, and H. A. Mooney. 1982. Endomycorrhizal role for interspecific transfer of phosphorus in a community of annual plants. Science 217:941-943.

Egerton-Warburton, L. M., J. I. Querejeta, and M. F. Allen. 2007. Common mycorrhizal networks provide a potential pathway for the transfer of hydraulically lifted water between plants. Journal of Experimental Botany 58:1473-1483. 
Gao, F. L., X. X. Che, F. H. Yu, and J. M. Li. 2019. Cascading effects of nitrogen, rhizobia and parasitism via a host plant. Flora 251:62-67.

Giovannetti, M., and B. Mosse. 1980. An evaluation of techniques for measuring vesicular arbuscular mycorrhizal infection in roots. New Phytologist 84:489-500.

Göransson, H., M. Welc, E. K. Bünemann, I. Christl, and H. O. Venterink. 2016. Nitrogen and phosphorus availability at early stages of soil development in the Damma glacier forefield, Switzerland; implications for establishment of $\mathrm{N}_{2}$-fixing plants. Plant and Soil 404:251-261.

Grime, J. P., J. M. L. Mackey, S. H. Hillier, and D. J. Read. 1987. Floristic diversity in a model system using experimental microcosms. Nature 328:420-422.

Haan, N. L., J. D. Bakker, and M. D. Bowers. 2018. Hemiparasites can transmit indirect effects from their host plants to herbivores. Ecology 99:399-410.

Hartley, S. E., J. P. Green, F. P. Massey, M. C. P. Press, A. J. A. Stewart, and E. A. John. 2015. Hemiparasitic plant impacts animal and plant communities across four trophic levels. Ecology 96:2408-2416.

Hu, S., F. S. Chapin, M. K. Firestone, C. B. Field, and N. R. Chiariello. 2001. Nitrogen limitation of microbial decomposition in a grassland under elevated $\mathrm{CO}_{2}$. Nature 409:188-191.

Jiang, Y., et al. 2017. Plants transfer lipids to sustain colonization by mutualistic mycorrhizal and parasitic fungi. Science 356:1172-1175.

Kiers, E. T., et al. 2011. Reciprocal rewards stabilize cooperation in the mycorrhizal symbiosis. Science 333:880-882.

Lekberg, Y., E. C. Hammer, and P. A. Olsson. 2010. Plants as resource islands and storage units-adopting the mycocentric view of arbuscular mycorrhizal networks. FEMS Microbiology Ecology 74:336-345.

Li, A. R., S. E. Smith, F. A. Smith, and K. Y. Guan. 2012. Inoculation with arbuscular mycorrhizal fungi suppresses initiation of haustoria in the root hemiparasite Pedicularis tricolor. Annals of Botany 109:1075-1080.

Li, J. M., Z. X. Jin, F. Hagedorn, and M. H. Li. 2014. Shortterm parasite-infection alters already the biomass, activity and functional diversity of soil microbial communities. Scientific Reports 4:6895.

Li, J. M., A. M. O. Oduor, F. H. Yu, and M. Dong. 2019. A native parasitic plant and soil microorganisms facilitate a native plant co-occurrence with an invasive plant. Ecology and Evolution 9:8652-8663.

Li, J. M., Z. C. Zhong, and M. Dong. 2008. Change of soil microbial biomass and enzyme activities in the community invaded by Mikania micrantha, due to Cuscuta campestris parasitizing the invader. Acta Ecologica Sinica 28:868-876.

March, W. A., and D. M. Watson. 2007. Parasites boost productivity: effects of mistletoe on litterfall dynamics in a temperate Australian forest. Oecologia 154:339-347.

Merrild, M. P., P. Ambus, S. Rosendahl, and I. Jakobsen. 2013. Common arbuscular mycorrhizal networks amplify competition for phosphorus between seedlings and established plants. New Phytologist 200:229-240.

Mikkelsen, B. L., S. Rosendahl, and I. Jakobsen. 2008. Underground resource allocation between individual networks of mycorrhizal fungi. New Phytologist 180:890-898.
Moora, M., et al. 2011. Alien plants associate with widespread generalist arbuscular mycorrhizal fungal taxa: evidence from a continental-scale study using massively parallel 454 sequencing. Journal of Biogeography 38:1305-1317.

Pinheiro, J., D. Bates, S. DebRoy, D. Sarkar, and R Core Team. 2018. nlme: linear and nonlinear mixed effects models. $\mathrm{R}$ package version 3.1-137. https://CRAN.R-project.org/packa ge $=$ nlme

Poulin, R. 2010. Network analysis shining light on parasite ecology and diversity. Trends in Parasitology 26:492-498.

R Development Core Team. 2018. R: a language and environment for statistical computing. R Foundation for Statistical Computing, Vienna, Austria. www.R-project.org/

Shi, Y. S., P. B. Zhao, K. Feng, A. M. Wang, and Y. W. Li. 2007. The occurrence of pests and diseases of white clover and its control measures. Plant Protection 32:70-73.

Sui, X. L., A. R. Li, Y. Chen, K. Y. Guan, L. Zhuo, and Y. Y. Liu. 2014. Arbuscular mycorrhizal fungi: potential biocontrol agents against the damaging root hemiparasite Pedicularis kansuensis? Mycorrhiza 24:187-195.

Sui, X. L., T. Zhang, Y. Q. Tian, R. J. Xue, and A. R. Li. 2019. A neglected alliance in battles against parasitic plants: arbuscular mycorrhizal and rhizobial symbioses alleviate damage to a legume host by root hemiparasitic Pedicularis species. New Phytologist 221:470-481.

Tesitel, J., J. Leps, M. Vrablova, and D. D. Cameron. 2011. The role of heterotrophic carbon acquisition by the hemiparasitic plant Rhinanthus alectorolophus in seedling establishment in natural communities: a physiological perspective. New Phytologist 192:188-199.

Walder, F., H. Niemann, M. Natarajan, M. F. Lehmann, T. Boller, and A. Wiemken. 2012. Mycorrhizal networks: common goods of plants shared under unequal terms of trade. Plant Physiology 159:789-797.

Watson, D. M. 2009. Parasitic plants as facilitators: more Dryad than Dracula? Journal of Ecology 97:1151-1159.

Weremijewicz, J., L. da Silveira Lobo O'Reilly Sternberg, D. P. Janos. 2018. Arbuscular common mycorrhizal networks mediate intra- and interspecific interactions of two prairie grasses. Mycorrhiza 28:71-83.

Weremijewicz, J., and D. P. Janos. 2013. Common mycorrhizal networks amplify size inequality in Andropogon gerardii monocultures. New Phytologist 198:203-213.

Weremijewicz, J., L. S. L. O. Sternberg, and D. P. Janos. 2016. Common mycorrhizal networks amplify competition by preferential mineral nutrient allocation to large host plants. New Phytologist 212:461-471.

Westwood, J. H., and G. Kim. 2017. RNA mobility in parasitic plant-host interactions. RNA Biology 214:450-455.

Workman, R. E., and M. B. Cruzan. 2016. Common mycelial networks impact competition in an invasive grass. American Journal of Botany 103:1041-1049.

Yang, B. F., X. Zhang, L. Zagorchev, J. M. Li, B. Frey, and M. H. Li. 2019. Parasitism changes rhizospheric soil microbial communities of invasive Alternanthera philoxeroides, benefitting the growth of neighboring plants. Applied Soil Ecology 143:1-9.

Zhang, J., J. M. Li, and M. Yan. 2013. Effects of nutrients on the growth of the parasitic plant Cuscuta australis $\mathrm{R}$.Br. Acta Ecologica Sinica 33:2623-2631. 